\title{
A community programme to reduce salt intake and blood pressure in Ghana [ISRCTN88789643]
}

\author{
Francesco P Cappuccio*1, Sally M Kerry², Frank B Micah³, Jacob Plange- \\ Rhule $^{3}$ and John B Eastwood ${ }^{4}$
}

Address: ${ }^{1}$ Clinical Sciences Research Institute, Warwick Medical School, UHCW Campus, Clifford Bridge Road, Coventry CV2 2DX, UK, ${ }^{2}$ Division of Community Health Sciences, St George's University of London, SW17 ORE, UK, ${ }^{3}$ Komfo Anokye Teaching Hospital, Kumasi, Ghana and ${ }^{4}$ Division of Cellular \& Molecular Medicine, St George's University of London, SW17 0RE, UK

Email: Francesco P Cappuccio* - F.P.Cappuccio@warwick.ac.uk; Sally M Kerry - s.kerry@sgul.ac.uk; Frank B Micah - fmicah@sgul.ac.uk; Jacob Plange-Rhule - jprhule@africaonline.com.gh; John B Eastwood - jbeastwo@sgul.ac.uk

* Corresponding author

Published: 24 January 2006

BMC Public Health 2006, 6:13 doi:10.1 186/147|-2458-6-13
Received: 21 July 2005

Accepted: 24 January 2006

This article is available from: http://www.biomedcentral.com//47/ $-2458 / 6 / 13$

(c) 2006 Cappuccio et al; licensee BioMed Central Ltd.

This is an Open Access article distributed under the terms of the Creative Commons Attribution License (http://creativecommons.org/licenses/by/2.0), which permits unrestricted use, distribution, and reproduction in any medium, provided the original work is properly cited.

\begin{abstract}
Background: In Africa hypertension is common and stroke is increasing. Detection, treatment and control of high blood pressure (BP) is limited. BP can be lowered by reducing salt intake. In Africa salt is added to the food by the consumer, as processed food is rare. A population-wide approach with programmes based on health education and promotion is thus possible.

Methods: We carried out a community-based cluster randomised trial of health promotion in I,0I3 participants from 12 villages (628 women, $48 \mathrm{I}$ rural dwellers); mean age 55 years to reduce salt intake and BP. Average BP was $125 / 74 \mathrm{mmHg}$ and urinary sodium (UNa) $10 \mathrm{l} \mathrm{mmol} / \mathrm{day}$. A health promotion intervention was provided over 6 months to all villages. Assessments were made at 3 and 6 months. Primary end-points were urinary sodium excretion and BP levels.
\end{abstract}

Results: There was a significant positive relationship between salt intake and both systolic (2.17 $\mathrm{mmHg}$ [95\% Cl 0.44 to $3.9 \mathrm{I}]$ per $50 \mathrm{mmol}$ of $\mathrm{UNa}$ per day, $\mathrm{p}<0.00 \mathrm{I})$ and diastolic $\mathrm{BP}(\mathrm{I} .10 \mathrm{mmHg}$ [0.08 to I.94], $\mathrm{p}<0.00 \mathrm{I})$ at baseline. At six months the intervention group showed a reduction in systolic ( $2.54 \mathrm{mmHg}$ [-I.45 to 6.54$])$ and diastolic $(3.95 \mathrm{mmHg}$ [0.78 to $7.1 \mathrm{I}], \mathrm{p}=0.015) \mathrm{BP}$ when compared to control. There was no significant change in UNa. Smaller villages showed greater reductions in $U N a$ than larger villages $(p=0.042)$. Irrespective of randomisation, there was a consistent and significant relationship between change in UNa and change in systolic BP, when adjusted for confounders. A difference in 24-hour UNa of $50 \mathrm{mmol}$ was associated with a lower systolic BP of $2.12 \mathrm{mmHg}$ (I.03 to $3.2 \mathrm{I})$ at 3 months and $1.34 \mathrm{mmHg}(0.08$ to 2.60$)$ at 6 months (both $\mathrm{p}<0.00 \mathrm{I}$ ).

Conclusion: In West Africa the lower the salt intake, the lower the BP. It would appear that a reduction in the average salt intake in the whole community may lead to a small but significant reduction in population systolic $B P$. 


\section{Background}

Non-communicable diseases are an important threat to the health of adults in Africa [1]. Worldwide, stroke is second only to ischaemic heart disease as a cause of death (over 4 million in 1990), and most of these deaths are in developing countries [2,3]. In sub-Saharan Africa, hypertension is common [4-7] and its detection, prevention, management and control should now be regarded as a priority [8]. It is estimated that if the 10-20 million people believed to have hypertension in sub-Saharan Africa were treated, about a quarter of a million deaths and twice as many long-term disabilities would be prevented annually [8]. Furthermore, in Africa, the reduction in population attributable risk when blood pressure (BP) is lowered is 13 times greater than in the USA [8]. However, where health-care provision is poor, detection and management of hypertension can only be haphazard and unreliable. Population-wide strategies to reduce BP could have an important impact on the number of strokes in the community. Whilst there is some data from Ghana using hospital series that stroke rates are increasing [9-11], one of the general problems in sub-Saharan Africa is the lack of reliable population-based morbidity and mortality data on stroke and other chronic diseases. Death certification and causes of death are not consistently recorded and cause-specific mortality rates are derived through verbal autopsies. Recent data from Tanzania [12] and South Africa [13] suggest a high burden of stroke, comparable to those seen in developed countries.

The World Health Organization regards population-wide strategies as an integral part of the overall approach to the prevention of cardiovascular disease worldwide, especially those that include dietary modification [14,15]. There is good evidence that a reduction in salt intake reduces $\mathrm{BP}$ [16] and that people of black African origin living in Africa respond well [17-19]. In the western world, it is very difficult to implement successful salt reduction strategies in the population since most of the salt ingested is in processed food. So, any intervention would involve the participation of the food industry and some attempts have been made in Portugal [20] and Finland [21]. In contrast, in populations whose intake of processed food is negligible - such as are found in many areas of sub-Saharan Africa - salt reduction strategies should be relatively easy to implement and have a good chance of success. To date, however, there have been no such community intervention studies in sub-Saharan Africa so the feasibility and effectiveness of a health education approach to BP reduction is unknown.

We started a programme with a view to establishing the feasibility of salt reduction as a way of reducing BP in twelve rural and semi-urban villages in the Ashanti region of Ghana where we suspect that hypertensive-related vas- cular diseases (namely stroke, renal and heart failure) account for a significant proportion of morbidity and mortality [22]. We conducted a pilot study, a household survey and population census, a survey of risk factors, subsequently randomised villages into clusters to receive general health education or more specific education as to how to reduce salt intake, then followed up the participants for six months to evaluate the effect on sodium excretion and BP. This paper describes the whole programme, highlights the strengths and limitations of our approach as well as the lessons learnt from our work, and suggests an interpretation in the context of the low-resource countries of subSaharan Africa.

\section{Methods \\ Ethics}

The study protocol was approved by the Committee on Human Research Publication and Ethics of the School of Medical Sciences, Kwame Nkrumah University of Science and Technology, Kumasi and endorsed by the St George's Healthcare NHS Trust Local Ethics Committee. As the study got under way meetings were held with the Chief and the Council of Elders in each village to explain the study and to seek their permission for the study to take place in their village.

\section{Study area and population}

The study was undertaken in the Ashanti region of central Ghana where the Disability Adjusted Life Expectancy (DALE) is 45.5 years [23]. Our study encompasses 12 communities in the Ejisu-Juabeng and Kumasi Districts [24]. The villages were chosen in consultation with local health workers [24]. The villages had little day-to-day contact and direct communication with each other [24], and transport between the villages is not generally available. Six villages were considered to be rural as they lack electricity and piped water, have a small population and are some distance from Kumasi [24,25]. The main occupation is farming. The remaining six villages were considered semi-urban. They are closer to Kumasi and usually have electricity and piped water [24,25]. Although the main activity is farming, trading is a frequent occupation. The Komfo Anokye Teaching Hospital, the only Tertiary Referral Hospital in the region, serves the area. In 1995 the hospital set up a BP clinic that now sees around 500 new patients a year [22].

\section{Household survey and population census}

Following agreement with the Chiefs and Elders in each village, a team of fieldworkers, fluent in both English and Twi (the local language), carried out a population census between January and March 2001 in order to create ageand-sex registers for the villages [24]. A total of 16,965 individuals (6,597 rural and 10,368 semi-urban) from 1,460 households (750 rural and 710 semi-urban) were 


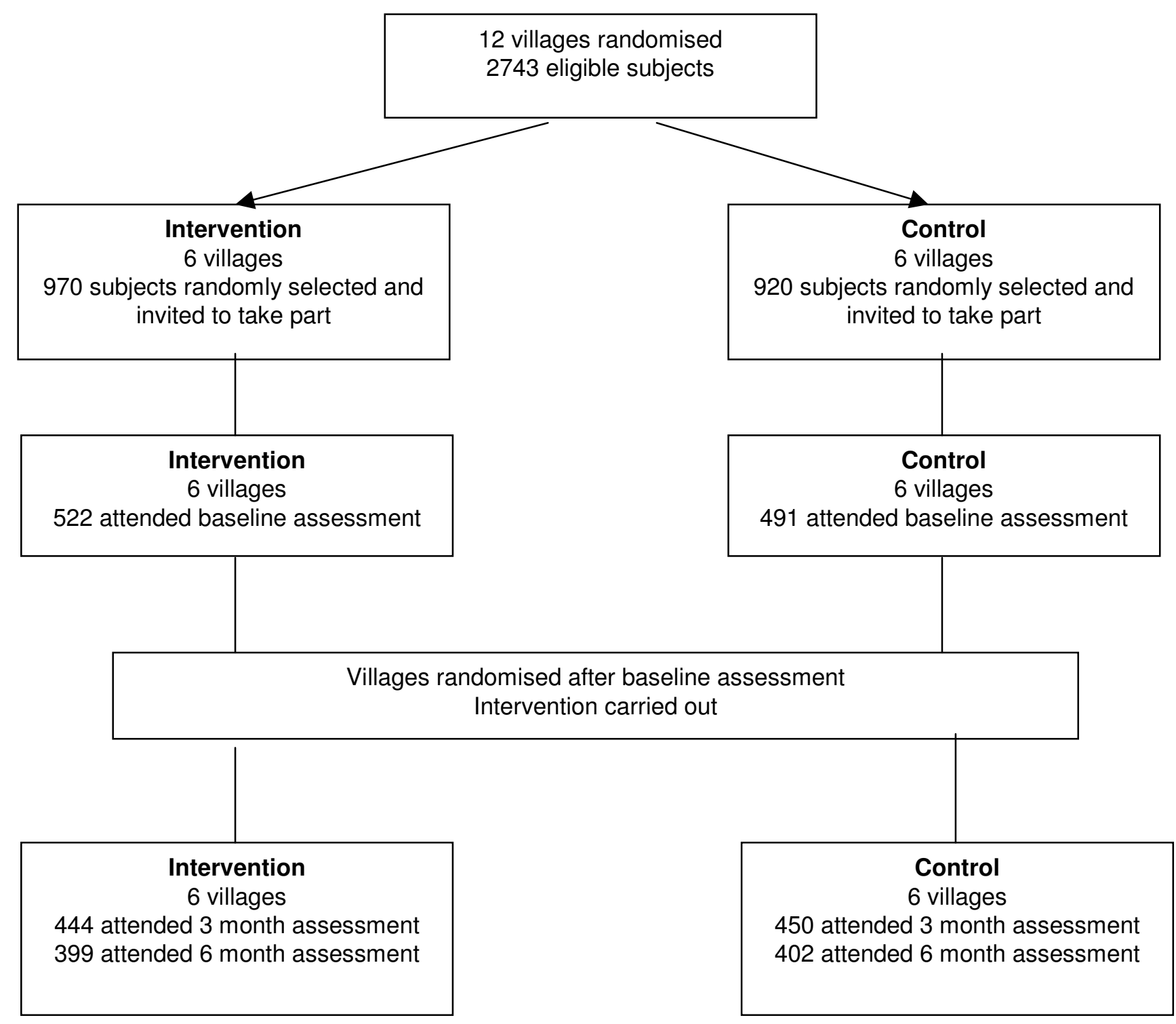

Figure I

Flow chart of the trial.

identified. The 12 villages had an average population (all ages) of 1,414 inhabitants (range 562-1,966) [24]. The adult population ( $>16$ years) was $50 \%$ in rural and $57 \%$ in semi-urban villages. The population structure of these villages was comparable to that reported for Ghana as a whole. There were 2,743 adults aged $40-75$ years.

\section{Recruitment of participants}

Between 95 and 250 subjects aged 40 to 75 from each village, total 1,896 , were selected by stratified random sampling by age and sex from the census of all inhabitants in the village so that the total sample selected matched the overall population structure. Between June 2001 and June
2002 a multidisciplinary study team set up a data collection centre in each village. All members of the team had been trained to take measurements and to carry out procedures while adhering to a protocol. The information sheet and consent form (available in both English and Twi) was explained to each participant who, if agreeing to take part, gave consent either by signing their name or applying a thumbprint.

\section{Baseline assessment}

For each consenting participant a fieldworker completed a detailed questionnaire, and measured height, weight and $\mathrm{BP}$. Where necessary the questioning was in Twi after local 
Table I: Regression coefficients (and 95\% C.I.) for blood pressure ${ }^{\pi}$ over sodium excretion adjusted for age, sex, locality, body mass index (random effect model)

\begin{tabular}{lll}
\hline Unit change & Systolic B.P. $(\mathbf{m m H g})$ & Diastolic B.P. (mmHg) \\
\hline 50 mmol of 24 h urinary sodium & $2.17(0.44 \text { to } 3.91)^{\#}$ & $1.01(0.08 \text { to } 1.94)^{\#}$ \\
Unit of sodium: creatinine ratio & $0.45(0.17 \text { to } 0.75)^{\#}$ & $0.18(0.02 \text { to } 0.33)^{\#}$ \\
Unit of sodium: potassium ratio & $1.74(0.45 \text { to } 3.03)^{\#}$ & $1.16(0.43 \text { to } 1.80)^{\#}$
\end{tabular}

I Adjusted for time of day. ${ }^{\#} \mathrm{p}<0.001$

verification of common expressions and translations. Socio-economic, lifestyle and medical information was collected. Information was also gathered on five salty foods - koobi, momoni, kako (all salted fish), salted pigs' feet and salted beef [25]. The respondents were asked if they ate these foods regularly and whether they added salt at the table or during cooking.

Height and weight were measured and body mass index (BMI) calculated [6]. BP and pulse rate were measured, and participants were also asked whether they were on regular anti-hypertensive drug therapy [6]. Venous blood was collected using vacutainers. Samples were transported to Komfo Anokye Teaching Hospital for aliquoting and storage [6]. Two consecutive timed 24-hour urine samples were obtained from each participant under close supervision. The urine samples were transported the same day to Komfo Anokye Teaching Hospital. Aliquots were prepared and frozen at $-20^{\circ} \mathrm{C}$. Serum and urine were later shipped to London on dry ice for long-term storage.

\section{Randomisation and power calculations}

After recruitment of participants, villages were allocated to intervention and control groups (Figure 1). Villages were randomised in blocks of two, and stratified for locality (semi-urban or rural) by an independent statistician [26]. Thus, intervention could begin in the first two villages while subjects in the second two were being recruited. The main outcomes of the trial were change in $24 \mathrm{~h}$ urinary sodium over the follow-up period and change in systolic BP. An average of 70 participants completing the trial from each village would allow a change in systolic BP of $4.8 \mathrm{mmHg}$ to be detected with $90 \%$ power, using a $5 \%$ significance level, assuming the standard deviation of the change in $\mathrm{BP}$ to be $12.6 \mathrm{mmHg}$ and the intra cluster correlation coefficient to be 0.02 [26]. Initially, 95 subjects were invited from each village to allow for a $25 \%$ refusal and attrition rate. As the response rate in the first four villages was lower than anticipated, more subjects were recruited in the remaining villages to obtain sufficient subjects overall and to allow for a greater refusal rate. The groups were comparable for baseline characteristics (Table 1).

\section{Intervention programme}

An intensive health education programme was carried out by community health workers. The educational and health promotion sessions were open to all villagers, irrespective of their participation into the trial. They were encouraged to attend by preliminary meetings with health visitors, the endorsement of their chief and community leaders. The meetings were held daily for the first week of the study and once weekly thereafter. The sessions were held in communal areas like churches, churchyards, schools, community centres. The sessions lasted approximately one hour (both for intervention and control). We did not record the actual number of attendants at each meeting as they were in relatively large numbers and, therefore, we are unable to report response rates at each session. The spirit of the intervention was to expose the whole community to the health promotion message and to test only a random proportion of them. A standard health education package from the Ghana Ministry of Health was used in all the villages. Flip charts were the main medium of communication; they consisted of double-sided A3-sized sheets with a colour picture on one side (shown to the participants) and written text on the other (facing the health visitor and used as a prompt). The public health issues included prevention of malaria, infective diarrhoeas and roundworm infection, as well as enhancing awareness of diabetes and high blood pressure. No mention was made of any possible dietary prevention of hypertension. In the intervention villages additional advice was given not to add salt to food and in cooking, to limit the amount of koobi, momoni, kako and tilapia (salted fish), salted pigs' feet and salted beef and to soak the items in water overnight before eating them. In order to avoid potential bias and contamination, we sought to maintain 'blindness' of the participants as to which dietary intervention they were receiving. On the other hand, we could not keep the community health nursing staff blind to the main objectives of the study. The feasibility of this approach had been tested in a small pilot study reported elsewhere [17].

Participants randomised to take part into the trial (both intervention and control) were given a small gift at the 
Table 2: Baseline characteristics of the population in the intervention and control groups.

\begin{tabular}{|c|c|c|}
\hline & Intervention $(\mathrm{n}=522)$ & Control $(n=49 I)$ \\
\hline Age & $54(\mathrm{II})$ & $55(\mathrm{II})$ \\
\hline Female n (\%) & $324(62)$ & $304(62)$ \\
\hline Height in $\mathrm{cm}$ & $160(8)$ & $161(8)$ \\
\hline Weight in $\mathrm{kg}$ & $54(\mathrm{II})$ & $54(\mathrm{II})$ \\
\hline Body mass index $\left(\mathrm{kg} / \mathrm{m}^{2}\right)$ & $21(4)$ & $21(4)$ \\
\hline Systolic blood pressure $(\mathrm{mmH}))^{\pi}$ & $129(25)$ & $127(27)$ \\
\hline Diastolic blood pressure $(\mathrm{mm} \mathrm{Hg})^{\pi}$ & $77(13)$ & $76(13)$ \\
\hline Heart rate $(\mathrm{bpm})^{\pi}$ & $74(12)$ & $72(12)$ \\
\hline Urinary sodium (mmol/24 h) & $99.9(44.7)$ & $102.5(45.3)$ \\
\hline Urinary potassium (mmol/24 h) & $47.5(23.2)$ & $49.3(23.6)$ \\
\hline Urinary creatinine (mmol/24 h) & $8.4(2.6)$ & $8.4(2.6)$ \\
\hline Urinary sodium/potassium ratio & $2.4(1.2)$ & $2.4(1.3)$ \\
\hline Urinary sodium/creatinine ratio & $12.4(5.3)$ & $12.7(5.6)$ \\
\hline Urinary potassium/creatinine ratio & $5.9(2.8)$ & $6.1(2.8)$ \\
\hline Current smoking n (\%) & $31(6)$ & $41(8)$ \\
\hline Alcohol drinking n (\%) & $199(38)$ & $211(43)$ \\
\hline Hypertensive $\mathrm{n}(\%)$ & $154(30)$ & $137(28)$ \\
\hline
\end{tabular}

2 subjects have missing urine collections at baseline

I Adjusted for time of day

Results are means (standard deviations) or number (percentage)

end of the study consisting of soap, rice, sugar, tinned mackerel, paracetamol, folic acid and some vitamin supplements; however they did not know this prior to participating, so that the response rate is likely to represent an unbiased response to a health promotion initiative. Likewise, the whole village was presented with a gift at the end of the study consisting of either a television set for the communal room (where there was electricity) or corrugated roofing sheets as a contribution to the building of local schools or community areas.

\section{Follow-up}

Participants were re-examined at 3 months and 6 months. At the 3-month visit a history of drug therapy was taken, and weight, BP and pulse were measured and recorded. Two 24-hour urine samples were collected but blood samples were not taken. At 6 months the same measurements were made, and on this occasion a blood sample as well. These urine and blood samples were processed in a similar way to the baseline samples. At 3 months 894 subjects attended and 889 (558 women and 331 men) were included in the analysis of urinary sodium. Of these 833 (531 women and 331 men) were based on two samples and 56 on one sample because a blinded assessment of the data indicated that one sample was incomplete or over-collected; $9 \%$ of men and $5 \%$ of women had only one sample. At 6 months 801 subjects attended and 800 (493 women and 307 men) were included in the analysis of urinary sodium. Of these 754 (465 women and 289 men) were based on two samples and 46 on one sample; $4 \%$ of men and $6 \%$ of women had only one sample. The overall response rate at baseline was 53\% $(1,013 / 1,896)$ but this varied considerably between villages (range $40 \%$ to $88 \%$ ). Response rates were higher in women than men; $58 \%(628 / 1,088)$ v $48 \%(385 / 808)$ and responders were 2.0 (95\% CI: 0.8 to 3.1 ) years older than non responders. In rural villages men and women responded similarly (57\% v 60\%; difference $-3 \%$ [-21 to 20\%]) but in the semi-urban villages men were less likely to respond $(41 \%$ v 56\%; difference $-15 \%$ [26 to $-4 \%]$ ). Follow-up rate at 6 months was similar in men and women $(307 / 385$ or $80 \%$ vs $494 / 628$ or $79 \%$ ). Attenders were 2.5 (0.8 to 4.2 ) years older than those who dropped out. Follow-up rates varied between villages from $62 \%$ to $92 \%$.

\section{Statistical analysis}

Regression analysis with robust standard errors was used to assess the effects of time of day on $\mathrm{BP}$ and pulse rate using the baseline measurements, adjusting for age, sex, locality, BMI and interaction between age and sex. All BP and pulse rate measurements were adjusted using these models prior to further analysis. The relationship between BP and sodium excretion, and between change in BP and change in sodium excretion were estimated using a random effects regression model with maximum likelihood estimation to allow for clustering within villages. The intervention effect was estimated as the difference in the change in the outcome in the intervention and control groups so that a negative difference favours the intervention. The analysis was carried out using a random effects regression. Adjusted changes in urine sodium-to-creatinine ratio for each village were estimated from ordinary least squares regression in each village and plotted using a random effect meta-analysis procedure. The correlation 

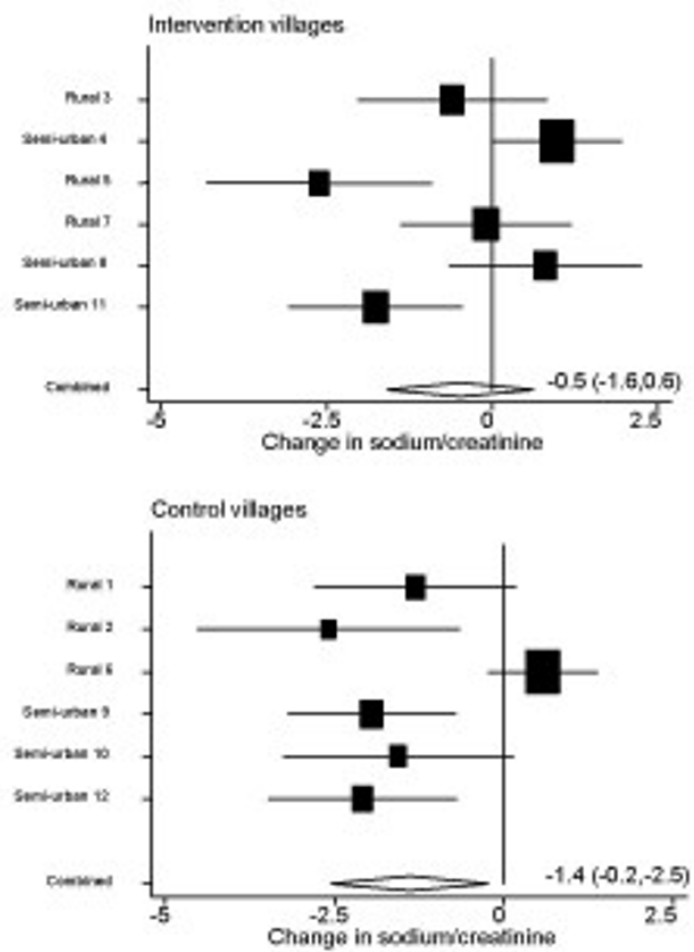

\section{Figure 2}

Changes in sodium-to-creatinine ratio adjusted for age, sex, $\mathrm{BMI}$ at baseline and locality (rural or semi-urban) in each village for the intervention and control groups of villages.

between the adjusted change in BP and the number of subjects was calculated using the meta-regression procedure. All analyses were carried out using Stata version 7.0.

\section{Results}

\section{General characteristics}

There were 1,013 participants in the study (385 men and 628 women); of these 532 were from semi-urban and 481 from rural villages. Ninety per cent of them reported being aged 40 to 75 years (mean age 54.7 years [SD 11.3]). Salt was almost invariably added to food in cooking (98\%) and salted foods such as fish and meat were eaten in both rural and semi-urban villages. Salt was often added at the table $(52 \%)$, more often in rural villages than in semiurban settings ( $59 \mathrm{v} 45 \%$; $\mathrm{p}<0.01$ ), although the total salt consumed as measured by urinary sodium was similar (99 v $103 \mathrm{mmol} /$ day). Urinary potassium levels were higher in rural villages (58 v $40 \mathrm{mmol} /$ day; difference $18 \mathrm{mmol} /$ day [11 to 26]; $\mathrm{p}<0.001)$. In semi-urban villages people were heavier (BMI 22.3 [4.6] vs 19.8 [3.2] kg/m2, p < $0.001)$ and had higher BP (129/76 [26/14] vs 121/72 [25/ 13] $\mathrm{mmHg}, \mathrm{p}<0.001$ for both systolic and diastolic BP).

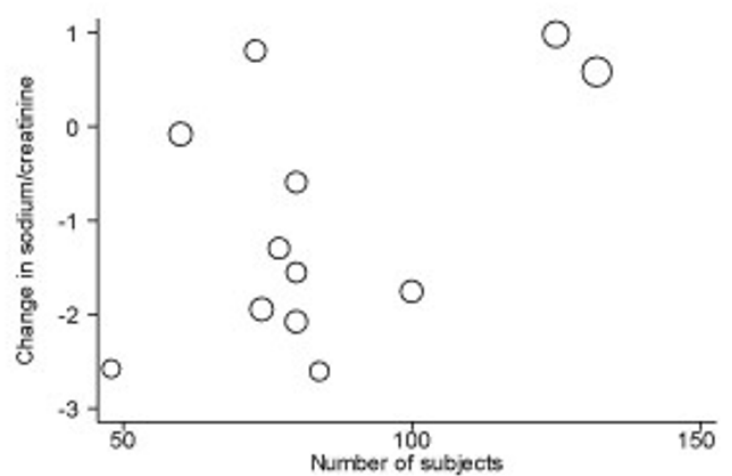

Figure 3

Changes in sodium-to-creatinine ratio adjusted for age, sex, BMI at baseline and locality by number of subjects recruited from each village. Regression coefficient $=-0.03[-0.05$ to 0$], P=0.042$ from meta-regression analysis.

The prevalence of hypertension increased with age and was more common in semi-urban settings [6].

\section{Effect of time of day}

There was a significant difference in the time of the day at which BP and pulse rate were taken at baseline (mean time of the day 9.28 [SD 1.39] equivalent to 9:17 am) compared with the two follow-up periods (7.57 [0.77] equivalent to $7: 34$ am at 3 months and 7.67 [0.73] equivalent to 7:40 am at 6 months), due to the necessity for taking much larger numbers of measurements at baseline. This difference in time of day of screening was associated with a significant drift in both BP and pulse rate. At baseline, there was a $1.90 \mathrm{mmHg}[95 \%$ CI 0.63 to 3.24 ; p < 0.001 ) lower systolic and a $1.45 \mathrm{mmHg}$ [0.66 to $2.20 ; \mathrm{p}<$ $0.001)$ lower diastolic BP for every hour of time of day of screening with a higher pulse rate of $0.85 \mathrm{bpm}$ per hour $(0.14$ to $1.56 ; \mathrm{p}<0.001)$. These estimates are compatible with those reported in earlier studies both in whites [27] and black Africans [28]. Further analyses are therefore reported with $\mathrm{BP}$ and pulse rate adjusted for time of day and standardised to 8:00 am.

\section{Relationship between urinary sodium and BP}

There was a significant and positive relationship between the level of salt intake (however estimated) and both systolic and diastolic BP (Table 1). When allowing for the potential confounding effects of age, gender, locality, BMI and clustering, there was a $2.2 \mathrm{mmHg}$ lower systolic and a $1.0 \mathrm{mmHg}$ lower diastolic BP for a $50 \mathrm{mmol} /$ day lower urinary sodium excretion. These relationships were signif- 


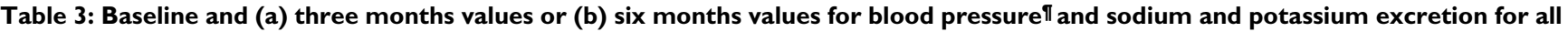
participants followed-up to that time point.

\begin{tabular}{|c|c|c|c|c|}
\hline \multirow[t]{2}{*}{ (a) } & \multicolumn{2}{|c|}{ Baseline } & \multicolumn{2}{|c|}{ Three months } \\
\hline & Intervention $(n=444)$ & Control $(n=450)$ & Intervention $(n=444)$ & Control $(n=450)$ \\
\hline Systolic blood pressure (mm Hg) & I28.5 (24.7) & $127.3(26.3)$ & $124.6(26.6)$ & $123.8(26.0)$ \\
\hline Diastolic blood pressure $(\mathrm{mm} \mathrm{Hg})$ & $76.8(13.0)$ & $75.8(13.5)$ & $74.2(13.7)$ & $74.0(14.1)$ \\
\hline Urinary sodium $(\mathrm{mm} / 24 \mathrm{~h})$ & $100.9(44.3)$ & $103.6(45.3)$ & $94.0(44.5)$ & $97.5(42.3)$ \\
\hline Urinary potassium $(\mathrm{mm} / 24 \mathrm{~h})$ & $48.3(23.5)$ & $49.3(23.4)$ & $50.1(20.8)$ & $52.1(23.6)$ \\
\hline Urinary creatinine $(\mathrm{mm} / 24 \mathrm{~h})$ & $8.4(2.6)$ & $8.4(2.6)$ & $7.8(2.5)$ & $7.9(2.8)$ \\
\hline Urinary sodium/potassium ratio & $2.4(1.2)$ & $2.4(1.3)$ & $2.1(1.1)$ & $2.1(1.1)$ \\
\hline Urinary sodium/creatinine ratio & $12.4(5.2)$ & $12.8(5.6)$ & $12.4(5.4)$ & $13.0(5.6)$ \\
\hline Urinary potassium/creatinine ratio & $6.0(2.8)$ & $6.1(2.8)$ & $6.7(2.9)$ & $6.9(2.9)$ \\
\hline \multirow[t]{2}{*}{ (b) } & \multicolumn{2}{|c|}{ Baseline } & \multicolumn{2}{|c|}{ Six months } \\
\hline & Intervention $(\mathrm{n}=399)$ & Control $(n=402)$ & Intervention $(n=399)$ & Control $(n=402)$ \\
\hline Systolic blood pressure $(\mathrm{mm} \mathrm{Hg})$ & $129.2(24.6)$ & $125.6(25.5)$ & $127.9(27.7)$ & $127.4(26.0)$ \\
\hline Diastolic blood pressure $(\mathrm{mm} \mathrm{Hg})$ & $76.9(13.0)$ & $75.2(13.3)$ & $76.0(14.2)$ & $78.7(14.3)$ \\
\hline Urinary sodium $(\mathrm{mm} / 24 \mathrm{~h})$ & $100.7(45.2)$ & $104.2(45.5)$ & $91.8(41.8)$ & $89.8(39.1)$ \\
\hline Urinary potassium $(\mathrm{mm} / 24 \mathrm{~h})$ & $48.5(23.9)$ & $50.9(24.2)$ & $48.2(21.5)$ & $52.1(21.9)$ \\
\hline Urinary creatinine $(\mathrm{mm} / 24 \mathrm{~h})$ & $8.4(2.6)$ & $8.4(2.5)$ & $8.0(2.6)$ & $8.0(2.5)$ \\
\hline Urinary sodium/potassium ratio & $2.4(1.3)$ & $2.4(1.3)$ & $2.2(1.2)$ & $1.9(1.0)$ \\
\hline Urinary sodium/creatinine ratio & $12.4(5.2)$ & $12.8(5.6)$ & $11.9(5.1)$ & II.7 (4.7) \\
\hline Urinary potassium/creatinine ratio & $6.0(2.9)$ & $6.3(2.9)$ & $6.3(2.7)$ & $6.8(2.7)$ \\
\hline
\end{tabular}

I Adjusted for time of day

Missing values for urinary variables; intervention, 3 months $n=1$, control, baseline $n=1,3$ months $n=4$

Missing values for urinary variables; control, baseline $n=I, 6$ months $n=1$

icant also when expressed as sodium-to-creatinine ratio and sodium-to-potassium ratio (Table 1).

\section{Effect of the intervention programme on salt intake}

The intervention and control groups were comparable for baseline characteristics (Table 2). In the intervention group $47 \%$ were from rural villages compared to $53 \%$ in the control group. During the health promotion programme the change in average sodium excretion varied between villages. It fell in four out of six villages in the intervention group and in 5 out of six villages in the control group (Figure 2). The net intervention effect was therefore a non-significant change in sodium excretion (Table 3 and 4). Smaller villages (with smaller samples) showed a greater reduction in urinary sodium excretion than larger villages (with larger samples) $(p=0.042)$ (Figure 3).

\section{Effect of the intervention programme on BP}

Intention-to-treat analysis

Throughout the trial we observed that the intervention group showed a small reduction in both systolic and diastolic BP, more pronounced at 6 months and statistically significant for diastolic BP at 6 months (Table 3 and
4). This effect was not consistent with the effect observed on urinary sodium excretion (Table 3 and 4 ).

\section{On-diet analysis}

At both 3 and 6 months, there was a consistent and significant relationship between the fall in urinary sodium excretion (however expressed) and the fall in systolic BP, when adjusting for confounders (Table 5). A $50 \mathrm{mmol}$ per day lower urinary sodium was associated with a fall in systolic BP between 1.0 and $3.2 \mathrm{mmHg}$ at 3 months and 0.1 to $2.6 \mathrm{mmHg}$ at 6 months. The effect on diastolic BP was less consistent, although in the same direction.

\section{Discussion}

This is the first attempt of a community-based trial of health promotion in rural and semi-urban villages in subSaharan Africa to achieve a population-wide reduction in salt intake with a view to reducing the population BP. The programme was complex and beset by practical difficulties. It has nevertheless produced important findings.

We have arrived at estimates of $\mathrm{BP}$ and salt intake in the Ashanti region of Ghana, and have gathered data on the efficacy and potential effectiveness of a programme of a 
Table 4: Effect of intervention (and $95 \% \mathrm{Cl}$ ) on reduction in blood pressure $\mathbb{I}^{\mathbb{l}}$ and urinary sodium excretion at 3 and 6 months adjusted for age, sex, locality, body mass index (random effect model)

\begin{tabular}{lcc}
\hline Variable & $\mathbf{3}$ months $(\mathbf{n}=\mathbf{8 9 4})$ & $\mathbf{6}$ months $(\mathbf{n}=\mathbf{8 0 I})$ \\
\hline SBP $(\mathrm{mmHg})$ & $-0.48(-5.45$ to 4.50$)$ & $-2.54(-6.54$ to 1.45$)$ \\
DBP $(\mathrm{mmHg})$ & $-1.02(-3.95$ to 1.91$)$ & $-3.95(-7.11 \text { to }-0.78)^{* *}$ \\
Urinary sodium $(\mathrm{mmol} / 24 \mathrm{~h})$ & $-0.5(-12.3$ to $1 \mathrm{l} .3)$ & $6.0(-4.1$ to 16.1$)$ \\
Urinary sodium: creatinine & $-0.01(-1.35$ to 1.32$)$ & $0.85(-0.53$ to 2.24$)$ \\
Urinary sodium: potassium & $0.08(-0.22$ to 0.39$)$ & $0.30(0.02 \text { to } 0.57)^{*}$
\end{tabular}

I Adjusted for time of day ${ }_{p}=0.03,{ }^{*} p=0.015$

Effect is control - intervention (negative values favour intervention).

modest reduction in salt intake in reducing the BP of African villagers. We chose the most difficult setting to implement a population-wide strategy, i.e. rural communities where the people are predominantly non-hypertensive and whose salt intake is lower than that found in most western countries.

\section{Strengths and limitations of our study}

To avoid potential confounding due to the localities we undertook stratification at cluster level. We also stratified at individual level in order to achieve the same age-gender structure of the invited sample of each village. Recruitment was carried out blind to avoid possible recruitment bias if the response rate proved to be low - as it was in some villages. The response rate was lower than expected for reasons unrelated to the intervention itself. There was a delay of up to 18 months between the household survey and starting the fieldwork in some villages. Villagers had sometimes forgotten that they had agreed to take part in the study and others had died or moved out of the area. Blood sampling was also sometimes a cause of difficulty. Blood was taken to measure factors related to cardiovascular disease but in some villages rumours spread that the blood was to be tested for HIV infection; in Ghana as in other parts of the world a positive HIV test carries a stigma for the whole family. Another rumour was that the blood would be sold in London. The timing of the blood sample required thought. While the time of data collection $(6 \mathrm{am}$ onwards) suited those working on their farms locally, as well as those remaining in the village, it did not suit those who left for work much earlier. This phenomenon proba- bly accounted for the lower response rate amongst men in semi-urban villages.

\section{Interpretation of our study}

Despite our careful planning, we did not manage to complete a fully randomised trial due to the contamination of the two intervention arms. Initially, we found the intervention effects difficult to interpret since the changes in $\mathrm{BP}$ and sodium excretion within each village were less than expected from the pilot study [17]. Possible explanations included contamination due to two reasons. First, the participants of intervention and control villages could have had contact with each other and exchanged information about the content of the health promotion. This was unlikely as the villages had been selected because not only were they several kilometres away from each other but also used different markets, and had no easy means of communication [24]. Alternatively, contamination could have arisen through the health workers who, despite their training and awareness of the purpose and scope of the study and the importance of concealing the true objectives, did unwittingly give information on salt reduction to villagers in the control villages in the belief that they should attempt to improve the health of all the villagers. Finally, a true variation in response between villages cannot be excluded. The disappointing outcomes could have been related to differing attitudes of different fieldworkers, variable co-operation of villagers (some villages were united, some less so), proportion of villagers in the village at the time of the educational sessions, and clashes with other events related to farming.

Table 5: Regression coefficients (and 95\% C.I.) for changes in blood pressurell for a given change in sodium excretion adjusted for age, sex, locality, body mass index (random effect model)

\begin{tabular}{lccc}
\hline Unit change & Month & Systolic B.P. (mmHg) & Diastolic B.P. (mmHg) \\
\hline 50 mmol of 24 h urinary sodium & 3 & $2.12(1.03 \text { to } 3.21)^{\#}$ & $1.11(0.45 \text { to } 1.78)^{\#}$ \\
Unit of sodium: creatinine ratio & 6 & $1.34(0.08 \text { to } 2.60)^{*}$ & $0.30(-0.45$ to 1.04$)$ \\
Unit of sodium: potassium ratio & 3 & $0.33(0.15 \text { to } 0.51)^{\#}$ & $0.14(0.03 \text { to } 0.25)^{* *}$ \\
& 6 & $0.30(0.08 \text { to } 0.53)^{\S}$ & $0.03(-0.10$ to 0.17$)$ \\
& 3 & $1.59(0.73 \text { to } 2.46)^{\#}$ & $0.48(-0.05$ to 1.00$)$ \\
\hline
\end{tabular}

I Adjusted for time of day ${ }^{\#} \mathrm{p}<0.001 ;{ }^{\S} \mathrm{p}=0.008 ;{ }^{*} \mathrm{p}=0.015 ;{ }^{*} \mathrm{p}=0.037$ 
If we accept that there was contamination - with some villages in the control group receiving advice on salt reduction, the regression analysis could provide an estimate of effect on BP of a reduction in urinary sodium excretion in all villages, irrespective of treatment allocation. We would then expect a greater reduction in BP for a greater reduction in urinary sodium. This is what we found, and it supports our concept. It can be argued, however, that this association can be merely due to some changes over time having an effect on both sodium and blood pressure. We cannot rule this out. We do not think our findings are explained by chance, though.

When measuring $\mathrm{BP}$, the possibility of confounding factors at village level cannot be ruled out, and we have shown, for example, that time of day is a significant predictor of BP. Since we have no records of ambient temperature, we have had to use time of day instead. The difference in $\mathrm{BP}$ predicted by a $2 \mathrm{~h}$ difference in the time of day is of the same order of magnitude of that expected by the intervention. Since all measurements were taken on the same day in each village, the $\mathrm{BP}$ measurements are also highly clustered. This means that in some villages, because of the effect of temperature, the $\mathrm{BP}$ may have risen even though sodium excretion may have fallen.

\section{Our data in the context of Africa}

Two intervention trials of dietary salt reduction in populations of African origin have been published so far $[18,19]$. Both were carried out in clinical settings under controlled experimental conditions and lasted 3-weeks. The authors suggest that the efficacy of a reduction in salt intake in developing countries equals that noted in more affluent cultures and in whites $[16,29]$. Our study extends these observations for the first time to a community setting and on a longer term to provide some evidence of effectiveness. As expected, the longer the study - in our case health promotion - the less pronounced the efficacy, presumably as a result of adaptation and reduced compliance as has been shown in studies in the Western world dealing with salt intake [30] and weight reduction [31].

Some have argued that health promotion programmes of cardiovascular prevention in the Western world should not be exported to developing countries because such programmes would be difficult to implement $[32,33]$. They have suggested that 'counting deaths' - a relatively easy task that focuses on 'surveillance' of cardiovascular disease in Africa - would lead to more effective prevention [32]. At the same time, they recognise that 'it is essential that context-appropriate health research and health interventions take place in developing countries' [33].

It would be simplistic to pre-judge the outcome of context-specific health promotion strategies based on experi- ences of the western world. Cultural and behavioural factors are often responsible for successes or failures of community interventions and it is likely that different mechanisms would operate in different contexts, so defeating mere generalisations. Furthermore, educational interventions in whole communities are 'complex interventions' that often pose significant problems of evaluation due to the difficulty of standardising their components [34].

Within the context of low-income countries in epidemiological transition, the case in favour of a population-based approach to limiting the rise in BP and ensuing stroke and renal risk is compelling [35,36]. In Ghana, structures for the detection, management and control of high BP outside cities and large towns are virtually absent [6]. Antihypertensive drugs are not free and most people in need cannot afford them [37]. It has been argued that drug treatment of cardiovascular risk factors in African communities will divert the already scarce resources away from needier areas of healthcare, such as maternal and child health, diarrhoeal diseases and malaria. The advent of free anti-retroviral therapy in many areas of sub-Saharan Africa will necessarily lead to the development of systems for the provision of drugs for the management of other chronic diseases. However, such advances are unlikely for many years, and the cheaper option of encouraging lifestyle changes is likely to be the preferred policy for some time.

\section{Perspectives}

In the DASH-Sodium study a reduction from 6.3 to $3.9 \mathrm{~g}$ of salt per day ( $\sim 38 \mathrm{mmol}$ of sodium per day) in nonhypertensive people caused a reduction in BP of 3.4/2.0 $\mathrm{mmHg}$ [38]. The effect was much greater in hypertensives, in African Americans and more pronounced the greater the change in salt intake $[16,38]$. By using the combined evidence from trials in the literature a modest reduction in salt intake of $\sim 3$ g per day ( $50 \mathrm{mmol}$ of sodium) in nonhypertensive people would reduce BP by 2 to $4 / 1$ to 2 $\mathrm{mmHg}$ [39]. This reduction, if sustained long-term, would be responsible for a reduction in stroke events of $12-14 \%$ per year [29,39]. The efficacy of salt reduction in lowering BP levels has been confirmed recently in two small shortterm clinical trials in Nigeria [18] and Jamaica [19].

How far would a reduction of $1.3 \mathrm{mmHg}$ in population systolic BP - as shown in our study - impact on stroke rates? No randomised clinical trials have been performed to show that a sustained reduction in salt intake leads to a reduction of cardiovascular events; however, evidence from prospective studies suggests that those eating less salt have a reduced risk of cardiovascular events [21,4042]. From prospective studies, randomised clinical trials of BP lowering treatment with drugs and short term trials 
of salt restriction it is estimated that stroke rates would fall by at least $10 \%$ in non-hypertensive individuals whose salt intake was reduced by $50 \mathrm{mmol}$ per day and systolic $\mathrm{BP}$ by $1.3 \mathrm{mmHg}$ [39]. In hypertensive individuals the benefit would be much greater. There is no evidence so far to guide us to extrapolate these benefits to African populations. It can be argued, however, that the attributable preventive fraction could be greater, given less competing cardiovascular risk in these populations.

Finally, our study design aimed specifically at changing salt intake, without targeting other aspects of the participants' diet. It is conceivable that a combined approach with an increase in potassium intake (not as high in this population as one would have expected) alongside a modification of their fat intake (mainly coming from the use of palm and coconut oils rather than dairy products) might exert compounding effects on lowering of blood pressure and prevention of strokes.

\section{Conclusion}

The impending epidemic of cardiovascular disease in subSaharan Africa in a context of lack of resources is a serious global public health challenge. Community-based strategies of health promotion for the management of chronic disease through lifestyle change in sub-Saharan Africa should be considered.

\section{Competing interests}

The author(s) declare that they have no competing interests.

\section{Authors' contributions}

FPC, SMK, JP-R and JBE contributed to fund raising, idea for and design of the study, collection of data, interpretation of results. FPC and SMK drafted the paper. FBM contributed to collection of data and interpretation of results. All authors contributed to the critical revision of the paper. FPC had full access to all the data in the study and takes responsibility for the integrity of the data and the accuracy of the data analysis

\section{Acknowledgements}

We thank the many workers in Ghana who have contributed to the study whose names are listed elsewhere [6]. We would also like to thank the Chiefs and Elders of the 12 villages for their help.

The study was supported by The Wellcome Trust (0604/5/Z/00/Z). FBM was supported by a Wellcome Trust Master's Research Training Fellowship (069500/Z/02/Z).

\section{References}

I. Cooper RS, Osotimehin B, Kaufman JS, Forrester T: Disease burden in sub-Saharan Africa: what should we conclude in the absence of data? Lancet 1998, 35 I:208-210.

2. Murray CJL, Lopez AD: Mortality by cause for eight regions of the world: Global Burden of Disease Study. Lancet 1997, 349: $1269-1276$.
3. Poungvarin N: Stroke in the developing world. Lancet 1998, 352(suppl III): 1922-1924.

4. Cooper RS, Rotimi C, Ataman S, McGee D, Osotomehin B, Kadiri S, Muna WFT, Kingue S, Fraser H, Forrester T, Bennet F, Wilks R: The prevalence of hypertension in seven populations of West African origin. Am J Public Health 1997, 87:160-168.

5. Amoah AGB: Hypertension in Ghana: a cross-sectional community prevalence study in Greater Accra. Ethn Dis 2003, 13:310-315.

6. Cappuccio FP, Micah FB, Emmett L, Kerry SM, Antwi S, Martin-Peprah R, Phillips RO, Plange-Rhule J, Eastwood JB: Prevalence, detection, management and control of hypertension in Ashanti, West Africa. Hypertension 2004:1017-1022.

7. Kearney PM, Whelton M, Reynolds K, Muntner P, Whelton PK, He J: Global burden of hypertension: analysis of worldwide data. Lancet 2005, 365:217-223.

8. Cooper RS, Rotimi C, Kaufman JS, Muna WFT, Mensah G: Hypertension treatment and control in sub-Saharan Africa: the epidemiological basis for policy. Br Med J 1998, 3 I 2:6 |4-617.

9. Anim JI, Kofi AD: Hypertension, cerebral vascular changes and stroke in Ghana: cerebral atherosclerosis and stroke. East African Medical Journal 1989, 66:468-475.

10. Nyame PK, Bonsu-Bruce N, Amoah AG, Adjei S, Nyarko E, Amuah EA, Biritwum RB: Current trends in the incidence of cerebrovascular accidents in Accra. West Afr Med J 1994, I 3: I 83- 186.

II. Wiredu EK, Nyame PK: Stroke-related mortality at Korle Bu Teaching Hospital, Accra, Ghana. East African Medical Journal 200I, 78: I80-I84.

12. Walker RW, McLarty DG, Kitange HM, Whiting D, Masuki G, Mtasiwa DM, Machibya H, Unwin N, Alberti KGMM: Stroke mortality in urban and rural Tanzania. Lancet 2000, 355: I684- 1687.

13. The SASPI Project Team: Prevalence of stroke survivors in rural South Africa. Stroke 2004, 35:627-632.

14. Report of a WHO/FAO Expert Consultation: Diet, nutrition and the prevention of chronic diseases. Volume 9/6. Geneva, World Health Organization. WHO Technical Report Series.; 2003:I-149.

15. World Health Organization: The World Health Report 2002. Reducing risks, promoting healthy life. Geneva, World Health Organization; 2002:1-248.

16. Sacks FM, Svetkey LP, Vollmer WM, Appel LJ, Bray GA, Harsha D, Obarzanek E, Conlin PR, Miller ER III, Simons-Morton DG, Karanja N, Lin P-H, for the DASH-Sodium Collaborative Research Group: Effects on blood pressure of reduced dietary sodium and the Dietary Approaches to Stop Hypertension (DASH) diet. $N$ Engl J Med 200I, 344:3-10.

17. Cappuccio FP, Plange-Rhule J, Phillips RO, Eastwood JB: Prevention of hypertension and stroke in Africa. Lancet 2000, 356:677-678.

18. Adeyemo AA, Prewitt TE, Luke A, Omotade OO, Rotimi CN, Brieger WR, Cooper RS: The feasibility of implementing a dietary sodium reduction intervention among free-living normotensive individuals in south West Nigeria. Ethn Dis 2002, | 2:207-2 | 2 .

19. Forrester T, Adeyemo A, Soarres-Wynter S, Sargent L, Bennett $F$, Wilks R, Luke A, Prewitt E, Kramer H, Cooper RS: A randomized trial on sodium reduction in two developing countries. J Hum Hypert 2005, 19:55-60.

20. Forte JG, Miguel JMP, Miguel MJP, de Padua F, Rose G: Salt and blood pressure: a community trial. J Hum Hypert 1989, 3:179-184.

21. Tuomilehto J, Jousilahti P, Rastenyte D, Moltchanov V, Tanskanen A, Pietinen $P$, Nissinen A: Urinary sodium excretion and cardiovascular mortality in Finland: a prospective study. Lancet 200I, 357:848-85I.

22. Plange-Rhule J, Phillips R, Acheampong JW, Saggar-Malik AK, Cappuccio FP, Eastwood JB: Hypertension and renal failure in Kumasi, Ghana. J Hum Hypert 1999, 13:37-40.

23. Mathers CD, Sadan R, Salomon JA, Murray CJL, Lopez AD: Healthy life expectancy in 191 countries. Lancet 200I, 357:1685-169I.

24. Plange-Rhule J, Cappuccio FP, Emmett L, Kerry SM, Edmundson AR, Acheampong $D$, Eastwood JB: A community study of health promotion in rural West Africa: details of a household survey and population census. QJ Med 2002, 95:445-450.

25. Kerry SM, Emmett L, Micah FB, Martin-Peprah R, Antwi S, Phillips RO, Plange-Rhule J, Eastwood JB, Cappuccio FP: Rural and semi-urban differences in salt intake, and its dietary sources, in Ashanti, West Africa. Ethn Dis 2005, I5:33-39. 
26. Kerry SM, Cappuccio FP, Emmett L, Plange-Rhule J, Eastwood JB: Reducing selection bias in a cluster randomised trial in West African villages. Clinical Trials 2005, 2: I25-I29.

27. Woodhouse PR, Khaw KT, Plummer M: Seasonal variation of blood pressure and its relationship to ambient temperature in an elderly population. J Hypertens I993, I I:I 267-I 274.

28. Chifamba J, Mufunda J, Sigola LB, Matenga JA, Sparks HV: Effect of variation in environmental temperature on blood pressure: is it important? Central Afr J Med 1998, 44:37-40.

29. He FJ, MacGregor GA: Effect of modest salt reduction on blood pressure: a meta-analysis of randomized trials. Implications for public health. I Hum Hypert 2002, 16:76 |-770.

30. Kumanyika SK, Cook NR, Cutler JA, Belden L, Brewer A, Cohen JD, Hebert PR, Lasser VI, Raines J, Raczynski J, Shepek L, Diller L, Whelton PK, Yamamoto M: Sodium reduction for hypertension prevention in overweight adults: further results from the Trials of Hypertension Prevention Phase II. J Hum Hypertens 2005, 1 9:33-45.

31. Dansinger ML, Gleason JA, Griffith JL, Selker HP, Schaefer EJ: Comparison of the Atkins, Ornish, Weight Watchers, and Zone diets for weight loss and heart disease risk reduction: a randomized trial. JAMA 2005, 293:43-53.

32. Ebrahim S: There are no shortcuts to finding out what works - population laboratories are essential tools. Tropical Medicine and International Health 1998, 3:256-257.

33. Ebrahim S, Davey-Smith G: Exporting failure? Coronary heart disease and stroke in developing countries. Int J Epidemiol 200 I, 30:20I-205

34. Hawe P, Shiell A, Riley T: Complex interventions: how "out of control" can a randomised controlled trial be? Br Med J 2004, 328: $|56|-1563$

35. Murray CJL, Lauer JA, Hutubessy RCW, Niessen L, Tomijima N, Rodgers A, Lawes CMM, Evans DB: Effectiveness and costs of interventions to lower systolic blood pressure and cholesterol: a global and regional analysis on reduction of cardiovascular-disease risk. Lancet 2003, 36 I:7I 7-725.

36. Rodgers A, Ezzati M, Vander HS, Lopez AD, Lin RB, Murray Cl: Distribution of Major Health Risks: Findings from the Global Burden of Disease Study. Plos Med 2004, I:e27.

37. Buabeng KO, Matowe L, Plange-Rhule J: Unaffordable drug prices: the major cause of non-compliance with hypertension medication in Ghana. J Pharm Pharmaceut Sci 2004, 7:350-352.

38. Bray GA, Vollmer WM, Sacks FM, Obarzanek E, Svetkey LP, Appel LJ: A further subgroup analysis of the effects of the DASH diet and three dietary sodium levels on blood pressure: results of the DASH-Sodium Trial. Am J Cardiol 2004, 94:222-227.

39. He FJ, MacGregor GA: How far should salt intake be reduced? Hypertension 2003, 42:1093-1099.

40. He J, Ogden LG, Vupputuri S, Bazzano LA, Loria C, Whelton PK: Dietary sodium intake and subsequent risk of cardiovascular disease in overweight adults. JAMA 1999, 282:2027-2034.

4I. He J, Whelton PK, Appel LJ, Charleston J, Klag MJ: Long-term effects of weight loss and dietary sodium reduction on incidence of hypertension. Hypertension 2000, 35:544-549.

42. Nagata C, Takatsuka N, Shimizu N, Shimizu H: Sodium intake and risk of death from stroke in Japanese men and women. Stroke 2004, 35: I543-I547.

\section{Pre-publication history}

The pre-publication history for this paper can be accessed here:

http://www.biomedcentral.com/1471-2458/6/13/prepub
Publish with Biomed Central and every scientist can read your work free of charge

"BioMed Central will be the most significant development for disseminating the results of biomedical research in our lifetime. "

Sir Paul Nurse, Cancer Research UK

Your research papers will be:

- available free of charge to the entire biomedical community

- peer reviewed and published immediately upon acceptance

- cited in PubMed and archived on PubMed Central

- yours - you keep the copyright

Submit your manuscript here:

http://www.biomedcentral.com/info/publishing_adv.asp
BioMedcentral 